The Astrophysical Journal, 488:94-108, 1997 October 10

(C) 1997. The American Astronomical Society. All rights reserved. Printed in U.S.A.

\title{
GAMMA-RAY PRODUCTION THROUGH INVERSE COMPTON SCATTERING WITH ANISOTROPIC PHOTON FIELD FROM ACCRETION DISK IN AGNs
}

\author{
L. ZhaNG AND K. S. CHENG \\ Department of Physics, University of Hong Kong, Pokfulam Road, Hong Kong \\ Received 1996 June 10; accepted 1997 May 22
}

\begin{abstract}
We give a detailed consideration of the photon spectrum produced by inverse Compton scattering off relativistic electrons in the anisotropic soft photon field from the accretion disk in jet model, assuming that the relativistic electrons and positrons are produced by collisions of relativistic protons with the soft photon and matter field. We considered soft photons from either the standard accretion disk or from the two-temperature accretion disk, respectively, which resulted in very different minimum Lorentz factors of the relativistic electrons and different scattered photon spectra. The scattered photon spectra seen from different angles and produced in various emission regions have been calculated for the different soft photon sources concerned and compared with results of other authors. The results of our calculations indicate that the instantaneous and stationary scattered photon spectra vary with the angle of scatter, the Lorentz factor of the jet, and the emission region of the $\gamma$-rays. Furthermore, whether X-rays and $\gamma$-rays can be produced cospatially depends upon the production mechanisms of the relativistic electrons. Subject headings: accretion, accretion disks - black hole physics - galaxies: active gamma rays: theory
\end{abstract}

\section{INTRODUCTION}

Fifty-one active galactic nuclei (AGNs) in high-energy regions $(E>100 \mathrm{MeV}$ ) have been detected by EGRET (von Montigny et al. 1995; Thompson et al. 1995). Various models have been proposed for explaining the $\gamma$-ray production. However, most models concern the idea that the $\gamma$-rays are produced by inverse Compton scattering off high-energy electrons in jet (e.g., Blandford \& Königl 1979), where soft photons can be provided by synchrotron radiation (synchrotron selfCompton model; Maraschi, Ghisellini, \& Celotti 1992), directly by a nearby accretion disk (e.g., Dermer, Schlickeiser, \& Mastichiadis 1992; Dermer \& Schlickeiser 1993 ), or by disk radiation scattered or reprocessed in some region of AGNs, e.g., line emission in the broad-line region (BLR), electron scattering in the BLR and intercloud medium, etc. (Sikora, Begelman, \& Rees 1994; Blandford \& Levinson 1995; Ghisellini \& Madau 1996). In these models the high-energy electrons are assumed to be isotropically distributed in the rest frame of the jet. Although the acceleration mechanisms of charged particles in AGNs are not yet clear, it is generally believed that positrons and electrons with very high energy can be produced in the collisions of relativistic protons with dense photon and matter fields and have a power-law distribution of energy (e.g., Sikora et al. 1987; Böttcher, Mause, \& Schlickeiser 1996).

The soft photons originating from the accretion disk can be divided into anisotropic and isotropic components. The anisotropic component comes directly from the accretion disk, and the isotropic component results from the disk photons reprocessed in some region (e.g., broad emission-line clouds). Obviously, it is important to estimate the relative importance of these two components in the production region of $\gamma$-rays in the jet. Based on the results of Protheroe, Mastichiadis, \& Dermer (1992), Bednarek, Kirk, \& Mastichiadis (1996) have estimated the sizes of the regions in which an anisotropic component dominates the inverse Compton energy loss of the electrons. Using reasonable parameters, the region can be up to $\sim 6000$ gravitational radii, which may be the region of $\gamma$-ray production (e.g., Dermer \& Schlickeiser 1994). Therefore, the calculation of inverse Compton scattering off the electrons in the anisotropic radiation field is necessary. Here we will adopt the standard accretion disk proposed by Shakura \& Sunyaev (1973) and the two-temperature accretion disk (e.g., Shapiro, Lightman, \& Eardley 1976) as two possible sources of the soft photons.

In the standard accretion disk, the radiation field depends on the surface temperature and on the photon spectrum. In principle, the distributions of surface temperature and local spectra of the thermal photons in different disk regions are different. However, as a good approximation, the disk can be considered to be radiating thermal photons as a blackbody with local temperature $T(R) \propto R^{-3 / 4}$. The two-temperature accretion disk, on the other hand, is divided into a hot region and a cool region. The temperature of electrons is almost constant and $T_{e} \sim 10^{9} \mathrm{~K}$ in the hot region, which is responsible for thermal X-ray emission. In the cool region, the surface temperature and local spectrum have the same distributions as those in the corresponding region in the standard accretion disk. The photon number density at any point above the accretion disk given by many authors (e.g., Bednarek 1993; Becker \& Kafatos 1995; Zhang \& Cheng 1997) is different from that used by Dermer \& Schlickeiser (1993) and Böttcher et al. (1996) (see § 2). In fact, the difference leads to different inverse Compton spectra, especially in the region near the central powerhouse, i.e., in the vicinity of the black hole (about 10 gravitational radii). In this paper the $\gamma$-ray spectra produced by inverse Compton scattering off the high-energy electrons in two different anisotropic radiation fields are calculated. In $\S 2$ the basic formulae for the calculations are given. The results for our model and a comparison with other results follow in $\S 3$, and a brief discussion is given in $\S 4$.

\section{INVERSE COMPTON SCATTERING}

We consider the $\gamma$-ray production in a simplified jet model shown schematically in Figure 1 . The jet is assumed to move 


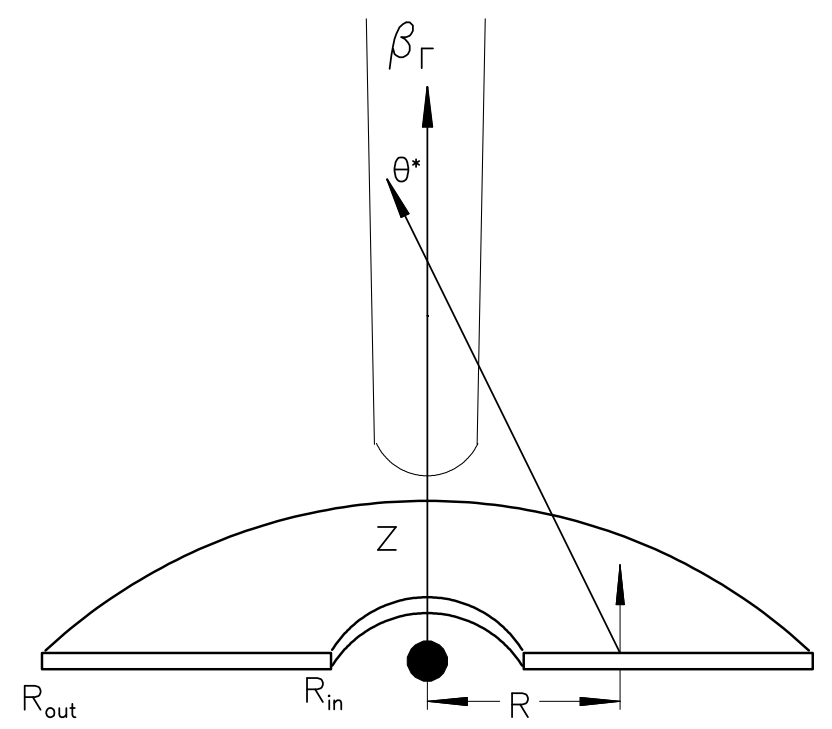

FIG. 1.-Schematic geometry of a relativistic AGN jet

outward vertically to the accretion disk plane with velocity $c \beta_{\Gamma}$ and Lorentz factor $\Gamma$. In this paper a superscript asterisk denotes physical quantities of photons measured in the rest frame of the accretion disk; quantities of photons without the superscript asterisk are measured in the rest frame of the jet; a subscript $s$ denotes the quantities of the scattered photon, and the photon energy is in units of rest energy of an electron, i.e., $\epsilon=h v / m_{e} c^{2}$.

In order to calculate the $\gamma$-ray production through inverse Compton scattering with the anisotropic photon field, we need to know the detailed expressions for the soft photon fields as well as the injected and evolution spectra of the relativistic electrons. If $n_{e}\left(\gamma, \Omega_{e}\right) d \gamma d \Omega_{e}$ represents the differential density of the electrons with Lorentz factor $\gamma$ between $\gamma+d \gamma$, which are directed inside solid-angle element $d \Omega_{e}$ in the direction $\Omega_{e}$, and $d^{2} n_{\mathrm{ph}} / d \epsilon d \Omega$ describes photon number density per unit solid angle at some distance to the central powerhouse, then the photon spectrum from inverse Compton scattering of the accretion disk photons in the rest frame of the jet can be expressed by (e.g., Dermer \& Schlickeiser 1993)

$$
\dot{n}_{\mathrm{ph}}\left(\epsilon_{s}, \Omega_{s}\right)=c \int_{0}^{\infty} d \epsilon \int d \Omega \frac{d^{2} n_{\mathrm{ph}}}{d \epsilon d \Omega} \int d \gamma \int d \Omega_{e}(1-\beta \cos \Phi) n_{e}\left(\gamma, \Omega_{e}\right) \frac{d^{2} \sigma}{d \epsilon_{s} d \Omega_{s}},
$$

where $d \sigma / d \epsilon_{s} d \Omega_{s}$ is the differential Compton scattering cross section in the rest frame of the jet, and

$$
\cos \Phi=\mu \mu_{e}+\left(1-\mu^{2}\right)^{1 / 2}\left(1-\mu_{e}^{2}\right)^{1 / 2} \cos \left(\phi-\phi_{e}\right) .
$$

In our calculation it is assumed that the injected spectrum of the electrons is isotropic in the rest frame of the jet. In general, the evolution of electron energy distribution is due to losses by synchrotron, inverse Compton scattering, bremsstrahlung, elastic scattering, and pair annihilation (see Böttcher et al. 1996) and gain through pair creation. For high-energy $\gamma$-ray production, the energy loss of the electron is dominated by inverse Compton scattering and/or synchrotron radiation. On the other hand, pair creation will be taken into account in the calculation of electron evolution if the optical depth due to pair production is larger than one. The optical depth in the anisotropic soft photon field from the accretion disk has been calculated (Becker \& Kafatos 1995; Zhang \& Cheng 1997); the results indicate that the optical depth has its minimum value along the axis of the accretion disk, i.e., $\gamma$-rays escape preferentially along the disk axis. In the small-angle regions around the axis, e.g., $\lesssim 15^{\circ}$ for $\mathrm{GeV} \gamma$-rays (Becker \& Kafatos 1995) and $\lesssim 7^{\circ}$ for TeV $\gamma$-rays (Zhang \& Cheng 1997), $\gamma$-rays can escape without significant decay caused by pair production within these angular ranges. Therefore, as an approximation, we can assume that the absorption of $\gamma$-rays can be neglected in the region of small angle between the emergent photons and disk axis in the $\gamma$-ray emission region considered. Here we only consider the inverse Compton energy loss of the electrons. Because the energy loss of the electrons depends on the radiation field and the radiation spectrum relates to electron distribution, the coupled equations for number densities of electrons and $\gamma$-rays have to be solved simultaneously in order to obtain a general $\gamma$-ray spectrum. However, if the injected spectrum of the electrons is a power law and the spectral index equals 2 , we show that the shape of the electron evolution spectrum does not change, although the energy range of the electron spectrum does. In this case the calculation of $\gamma$-ray production spectrum can be simplified.

\subsection{Photon Number Density from the Standard Accretion Disk}

In the standard accretion disk (Shakura \& Sunyaev 1973), the energy flux is

$$
Q=Q_{0} r^{-3} I(r) \text { ergs } \mathrm{cm}^{-2} \mathrm{~s}^{-1},
$$

where $Q_{0} \approx 6 \times 10^{16} M_{8}^{-2} \dot{M}_{26}, r=R / 10 R_{g}$ is the radius of the accretion disk in units of $10 R_{g}$, and $I(r)=\left(1-b r^{-1 / 2}\right)$, with $b=0.6^{1 / 2} \cdot M_{8}$ and $\dot{M}_{26}$ are the mass of the black hole in units of $10^{8} M_{\odot}$ and the mass accretion rate in units of $10^{26} \mathrm{~g} \mathrm{~s}^{-1}$, 
respectively. $R_{g}$ is the gravitational radius. Furthermore, the surface temperature of the accretion disk can be given by

$$
T(r)=T_{0} r^{-3 / 4} I^{1 / 4}(r) \mathrm{K},
$$

where $T_{0} \approx 10^{5} M_{8}^{-1 / 2} \dot{M}_{26}^{1 / 4}$. Here, the inner and outer radii of the accretion disk are $R_{\text {in }}=10 R_{g} r_{\text {in }}$ and $R_{\text {out }}=10 R_{g} r_{\text {out }}$, respectively. Assuming that the distribution of the disk photons $G(X)$ is the blackbody distribution

$$
G(X)=\frac{X^{3}}{\exp X-1}
$$

with $X=m_{e} c^{2} \epsilon^{*} / k T(r)$, the differential energy flux can be expressed by

$$
F_{\mathrm{ph}}=F_{0} I_{1}(r, X) \operatorname{ergs} \mathrm{cm}^{-2} \mathrm{~s}^{-1} \mathrm{ergs}^{-1},
$$

where $F_{0}=Q_{0} / k T_{0} C$ and $C=\int_{0}^{\infty} G(X) d X ; I_{1}=r^{-9 / 4} I^{3 / 4}(r) G(X)$. Now we would like to give the photon number density per unit solid angle per dimensionless energy of the photon at distance $z$ to the black hole. Referring to the geometry shown schematically in Figure 1, we assume that the $z$-axis is the axis of symmetry of the accretion disk; the relation between the solid angle $d \Omega^{*}$ and the emission area, $d A^{*}=R d R d \phi$, is given by

$$
d \Omega^{*}=\frac{z}{\left(z^{2}+R^{2}\right)^{3 / 2}} d A^{*}
$$

where the extra factor $z /\left(z^{2}+R^{2}\right)^{1 / 2}$ in equation (7) is the cosine angle $\left(\cos \theta^{*}\right)$ between the disk normal direction and the photon emission direction, and $\left(z^{2}+R^{2}\right)^{1 / 2}$ is the distance between the symmetry axis at height $z$ and the photon emission point in the accretion disk. After transforming from $R$ to $\theta^{*}$ by using $\tilde{z}=z / 10 R_{g}, \mu^{*}=\cos \theta^{*}$, and $r=\left(R / 10 R_{g}\right)=\tilde{z} \tan \theta^{*}=$ $\chi\left(\tilde{z}, \mu^{*}\right)$, the photon number density can be expressed as

$$
\left(\frac{d n_{\mathrm{ph}}^{2}}{d \epsilon d \Omega}\right)^{*}\left(\epsilon^{*}, \tilde{z}, \mu^{*}\right)=\frac{1}{2 \pi c \epsilon^{*}} F_{\mathrm{ph}}\left[\epsilon^{*}, \chi\left(\tilde{z}, \mu^{*}\right)\right]
$$

where the cosine of the angle between an incident photon and an electron, $\mu^{*}=\cos \theta^{*}$, is given by

$$
\mu^{*}=\frac{\tilde{z}}{\left(\tilde{z}^{2}+r^{2}\right)^{1 / 2}} .
$$

Turning to the rest frame of the jet, the relevant Lorentz transformation equations are $\epsilon^{*}=\Gamma \epsilon\left(1+\beta_{\gamma} \mu\right)$ and $\mu^{*}=$ $\left(\mu+\beta_{\Gamma}\right) /\left(1+\beta_{\Gamma} \mu\right)$. Because of azimuthal symmetry of the photon source, $d \Omega^{*}=2 \pi d \mu^{*}$. Therefore, the photon number density in the rest frame of the jet is given by using $\left(d^{2} n_{\mathrm{ph}} / d \epsilon d \Omega\right) / \epsilon^{2}=\left(d n_{\mathrm{ph}}^{* 2} / d \epsilon^{*} d \Omega^{*}\right) / \epsilon^{* 2}$ (Rybicki \& Lightman 1979),

$$
\frac{d^{2} n_{\mathrm{ph}}}{d \epsilon d \Omega}(\epsilon, \tilde{z}, \mu)=\Gamma^{-2}\left(1+\beta_{\Gamma} \mu\right)^{-2}\left(\frac{d^{2} n_{\mathrm{ph}}}{d \epsilon d \Omega}\right)^{*}\left(\epsilon^{*}, \tilde{z}, \mu^{*}\right),
$$

where $\mu=\cos \theta$.

\subsection{Photon Number Density from the Two-Temperature Accretion Disk}

In the two-temperature accretion disk (e.g., Shapiro et al. 1976; Eilek \& Kafatos 1983), the temperature of thermal electrons in the hot region is almost constant in the region $T_{e} \sim 10^{9}-10^{10} \mathrm{~K}$, by the Compton thermostat mechanism. When the Compton $y$-parameter $y \equiv\left(4 k T_{e} / m_{e} c^{2}\right) \max \left(\tau_{\mathrm{es}}, \tau_{\mathrm{es}}^{2}\right)>1$, these electrons in the hot region produce a power-law inverse Compton photon spectrum extending up to $k T_{e}$, at which point there is an exponential turnover by upscattering UV radiation produced in the cool surrounding region, where $\tau_{\text {es }}$ is the electron-scattering half-thickness of the disk (e.g., Svensson 1994). Therefore, at nonrelativistic temperatures $\left(T_{e} \lesssim 5 \times 10^{9} \mathrm{~K}\right)$ and for $\tau_{\mathrm{es}}>1$, the differential energy flux in the hot region from $R_{\mathrm{in}}$ to $R_{\mathrm{ho}}$ (outer radius of the hot region) can be approximated by

$$
F_{\mathrm{ph}}^{h}=F_{0}^{h} \epsilon^{*-\alpha} \exp \left(-\frac{m_{e} c^{2}}{k T_{e}} \epsilon^{*}\right) \operatorname{ergs} \mathrm{cm}^{-2} \mathrm{~s}^{-1} \mathrm{ergs}^{-1},
$$

where the spectral index $\alpha \approx-1.5+(2.25+4 / y)^{1 / 2}$ (e.g., Rybicki \& Lightman 1979). The exact solution of the Comptonization problem for the nonrelativistic case and diffusion region was first derived by Sunyaev \& Titarchuk (1980). Furthermore, the nonrelativistic solution was generalized for the subrelativistic case by Hua \& Titarchuk (1995). Here we assume that $T_{e}=10^{9} \mathrm{~K}$, so we can use approximating expressions at the nonrelativistic region. From equations (3) and (11), $F_{0}^{h}$ can be determined and

$$
F_{0}^{h}=\frac{Q_{0}}{m_{e} c^{2} A(\alpha)} r^{-3} I(r),
$$

where $A(\alpha)=\int d \epsilon^{*} \epsilon^{*-\alpha} \exp \left(-5.93 \epsilon^{*}\right)$. For the cool region from $R_{\mathrm{ho}}$ to $R_{\mathrm{out}}$, the differential energy flux is given by equation (6), that is,

$$
F_{\mathrm{ph}}^{c}=F_{0} I_{1}(r, X) .
$$


Therefore the total differential energy flux is the sum of equations (11) and (13). It can be written as

$$
F_{\mathrm{ph}}= \begin{cases}F_{0}^{h} \epsilon^{*-\alpha} \exp \left(-\frac{m_{e} c^{2}}{k T_{e}} \epsilon^{*}\right) H\left(\epsilon^{*}-\epsilon_{0}^{*}\right) & r_{\mathrm{in}} \leq r \leq r_{\mathrm{ho}}, \\ F_{0} I_{1}(r, X) & r_{\mathrm{ho}} \leq r \leq r_{\mathrm{out}},\end{cases}
$$

where $H(x)$ is the step function, and $\epsilon_{0}^{*}$ is the energy at which the above expression for the hot region is valid (Shapiro et al. 1976). Inserting equation (14) into equation (8), the photon number density per unit solid angle per dimensionless energy of the photon at the distance $z$ can be obtained for the two-temperature accretion disk.

In the two-temperature accretion model, it is necessary to determine $\alpha$ and $R_{\mathrm{ho}}$ initially. $\alpha$ generally depends upon the Compton $y$-parameter, and $\alpha \sim 1$ for $y \sim 1$. The typical value of $R_{\mathrm{ho}}$ is from $30 R_{g}$ to $100 R_{g}$ (see, e.g., Shapiro et al. 1976). In our calculations, we assume that $\alpha$ and $R_{\mathrm{ho}}$ are constants and let $\alpha=0.9, R_{\mathrm{ho}}=50 R_{g}$, and $m_{e} c^{2} \epsilon_{0}^{*} \approx 10 \mathrm{eV}$.

\subsection{The Distribution of the Relativistic Electrons}

It is generally believed that relativistic charged particles can be efficiently accelerated in the plasma near the black hole. Although acceleration mechanisms of charged particles in AGNs are not yet clear, a common idea is that accelerated primary electrons cannot obtain higher energy because of their serious energy loss. However, secondary positrons and electrons with very high energy can be produced in the collisions of relativistic protons with dense photon and material fields (i.e., in $p \gamma$ and $p p$ collisions) and have a power-law distribution if the accelerated protons have sufficiently high energy and power-law distribution (e.g., Sikora et al. 1987; Begelman, Rudak, \& Sikora 1990; Atoyan 1992; Böttcher et al. 1996). However, there are different lower limits for the secondary electron energy because of the threshold energies of the production processes of the above-mentioned secondary electrons. In the $p p$ collision, the lower limit of the electron Lorentz factor is given by

$$
\gamma_{\min }^{p p} \approx \frac{1}{24}\left[\left(E_{\mathrm{th}}-m_{p} c^{2}\right) / \mathrm{MeV}\right]
$$

with $E_{\mathrm{th}}=1.22 \mathrm{GeV}$ (e.g., Mannheim \& Schlickeiser 1994), i.e., $\gamma_{\min }^{p p} \approx 12$. If $p p$ collision dominates in some region of AGNs, then the minimum Lorentz factor of the electrons is given by $\gamma_{\mathrm{min}}^{p p}$. In the $p \gamma$ collision, the estimation of the lower limits of the electron Lorentz factor depends on the mean energy of the soft photons. For a given energy of soft photons, the minimum Lorentz factor of the electrons is given by the photopair production process (e.g., Mannheim \& Biermann 1989; Begelman et al. 1990; Chodorowski, Zdziarski, \& Sikora 1992) instead of by the higher threshold of photomeson production. Therefore the minimum Lorentz factor of the relativistic electrons is determined by

$$
\gamma_{\min }^{p \gamma} \approx \frac{m_{e} c^{2}}{\left\langle\epsilon^{*}\right\rangle},
$$

where $\left\langle\epsilon^{*}\right\rangle$ is the mean energy of the soft photons. For example, $\gamma_{\min }^{p \gamma} \sim 500-1000$ in the standard accretion disk proposed by Shakura and Sunyaev (Böttcher et al. 1996), which corresponds to soft photon mean energies of 100 and $50 \mathrm{eV}$, respectively. However, in the two-temperature accretion model (Shapiro et al. 1976), the mean energy of X-rays is about $100 \mathrm{keV}$, so the minimum Lorentz factor of the electrons is much smaller than that of the standard accretion disk and is about 5. Furthermore, Mrk 421 and Mrk 501 have been observed to emit $\gamma$-rays with TeV energy (Punch et al. 1992; Quinn et al. 1996); relativistic electrons of Lorentz factor greater than $10^{6}$ are required for explaining the observations. We assume, therefore, that the maximum Lorentz factor of the electrons is $10^{6}$, but the minimum Lorentz factor of the electrons is different for the standard accretion model and the two-temperature accretion model in our calculations. Furthermore, we assume that the differential density of the electrons is isotropic [i.e., $\left.n_{e}\left(\gamma_{i}, \Omega_{e}\right)=n_{e}\left(\gamma_{i}\right) / 4 \pi\right]$ in the rest frame of the jet, and

$$
n_{e}\left(\gamma_{i}\right)=n_{e}^{0} \gamma_{i}^{-s}, \quad \gamma_{\min }^{i}<\gamma_{i}<\gamma_{\max }^{i},
$$

where $\gamma_{\min }^{i}$ and $\gamma_{\max }^{i}$ are the minimum and maximum Lorentz factors of the electron, respectively.

Because of the energy loss of the relativistic electrons, the electron spectrum will evolve, resulting in changes of $\gamma_{\min }, \gamma_{\max }$, and the shape of the electron spectrum. Here we consider inverse Compton energy loss of the electrons. For simplicity, we consider a single electron energy loss rate resulting from inverse Compton scattering in the Thomson region. For a single electron, $n_{e}(\gamma)=\delta(\gamma-\bar{\gamma}) / 4 \pi$ in the jet frame. In the Thomson region, the average energy $\epsilon_{s}$ of scattered photons in the rest frame of the jet is about $\gamma^{2} \epsilon(1-\beta \cos \Phi)$, and these photons are beamed into a cone with half-angle $\theta_{s} \approx \gamma^{-1} \ll 1$ about the original direction of the electron's motion; then the differential cross section can be approximated by (Dermer $\&$ Schlickeiser 1993)

$$
\left(\frac{d^{2} \sigma}{d \epsilon_{s} d \Omega_{s}}\right)_{\mathrm{T}} \approx \sigma_{\mathrm{T}} \delta\left[\epsilon_{s}-\gamma^{2} \epsilon(1-\beta \cos \Phi)\right] \delta\left(\Omega_{s}-\Omega_{e}\right)
$$

Using the above expressions, the scattered photon number density can be given as

$$
\dot{n}_{\mathrm{ph}}=\frac{\sigma_{\mathrm{T}}}{4 \pi^{2} \Gamma^{2}} \gamma^{-2} \int d \Omega_{s}\left(1+\beta_{\Gamma} \mu\right)^{-2} \frac{F_{\mathrm{ph}}\left\{u, \chi\left[\tilde{z},\left(\mu+\beta_{\Gamma}\right) /\left(1+\beta_{\Gamma} \mu\right)\right]\right\}}{u},
$$

where $u=\Gamma \epsilon_{s}\left(1+\beta_{\Gamma} \mu\right) / \gamma^{2}\left(1-\beta_{\Gamma} \cos \Phi\right)$ and $\cos \Phi$ is given by equation (2) with $\mu_{e}=\mu_{s}$, and equations (1), (10), (8), and (18) have been used to derive equation (19). Therefore, from the energy loss rate of the inverse Compton scattering for an electron 
with Lorentz factor $\gamma$, at height $z$, and moving at angle $\mu_{s},-(d \gamma / d t)_{\mathrm{IC}}=\int_{0}^{\infty} d \epsilon_{s} \epsilon_{s} \dot{n}_{\mathrm{ph}}$, the electron equation of motion for a given angle $\mu_{s}^{*}$ is given by (see Appendix A)

$$
-\frac{d \gamma}{d \tilde{z}}=\xi U\left(\mu_{s}^{*}, \tilde{z}\right) \gamma^{2}
$$

Its solution is

$$
n_{e}\left(\gamma, \mu_{s}^{*}, \tilde{z}\right)=n_{e}^{0} \gamma^{-2}\left[\gamma^{-1}+\xi V\left(\mu_{s}^{*}, \tilde{z}\right)\right]^{s-2} \quad \gamma_{\min }\left(\mu_{s}^{*}, \tilde{z}\right)<\gamma<\gamma_{\max }\left(\mu_{s}^{*}, \tilde{z}\right),
$$

where $\gamma_{\min }$ and $\gamma_{\max }$ are minimum and maximum Lorentz factors of the evolving electron spectrum and can be determined by equation (A11), with $\gamma_{\min }\left(\tilde{z}_{i}\right)=\gamma_{\min }^{i}$ and $\gamma_{\max }\left(\tilde{z}_{i}\right)=\gamma_{\max }^{i}$, respectively. $U\left(\mu^{*}, \tilde{z}\right), \xi$ and $V\left(\mu_{s}^{*}, \tilde{z}\right)$ are given in equations (A2), (A5), and (A12) of Appendix A. It can be seen from equation (21) that the shape of the evolving electron spectrum is the same as that given by equation (17) if $s=2$. Finally, we would like to point out that the electron spectrum in the steady state is given by

$$
n_{\mathrm{es}}(\gamma)=\left(\frac{d \gamma}{d t}\right)^{-1} \int n_{e} \gamma d \gamma \propto \gamma^{-s-1}
$$

in which equations (17) and (A1) have been used.

\subsection{Photon Production Rate from Inverse Compton Scattering}

The treatment of inverse Compton scattering off the electrons has been investigated by many authors (e.g., Rieke \& Weekes 1969; Blumenthal \& Gould 1970; Reynolds 1982; Dermer \& Schlickeiser 1993; Böttcher et al. 1996). The photon spectrum from inverse Compton scattering of the accretion disk photons in the rest frame of the jet can be expressed by equations (1) and (2). It is convenient to use the approximations of the differential Compton cross section in Thomson and Klein-Nishina (KN) regions for calculating the photon spectrum. Here we use the approximations of the differential cross sections in these two regions given by Dermer \& Schlickeiser (1993), and we give the scattered photon spectra for the soft photons from the standard accretion disk and from the two-temperature accretion disk, respectively.

For the soft photons from the standard accretion disk, the distribution of the disk photons $G(X)$ is given by equation (5). In the rest frame of the standard accretion disk, using equations (4) and (6), and $r=\tilde{z}\left(1-\mu^{*}\right)^{1 / 2} / \mu^{*}$, the scattered photon spectrum in the Thomson region is given by (see Appendix B for details)

$$
\dot{n}_{\mathrm{ph}}^{\mathrm{T}}\left(\epsilon_{s}^{*}, \mu_{s}^{*}, \tilde{z}\right)=\frac{D \sigma_{\mathrm{T}} \dot{N}_{0}}{8 \pi^{2}}\left\{\int_{\max \left[\gamma_{\min }, \epsilon_{s}^{*} / D\right]}^{\gamma_{\max }} d \gamma \gamma^{-2} n_{e}\left(\gamma, \mu_{s}^{*}, \tilde{z}\right) \int_{\mu_{\min }}^{\mu_{\max }{ }^{*}} d \mu^{*} J_{1}\left(\mu^{*}, \tilde{z}\right)\left(\frac{\eta \gamma^{2}}{\epsilon_{s}^{*}}\right) G(X)\right\},
$$

where $J_{1}\left(\mu^{*}, \tilde{z}\right)$ and $J_{2}\left(\mu^{*} \tilde{z}\right)$ are given by equations (B6) and (B7) in Appendix B, $G(X)$ is given by equation (5), and

$$
X\left(\mu^{*}, \tilde{z}, \epsilon_{s}^{*}\right)=\frac{m_{e} c^{2}}{E_{0}} \frac{\epsilon_{s}^{*}}{\gamma^{2}} \frac{1}{J_{2}\left(\mu^{*}, \tilde{z}\right) \eta}
$$

where $E_{0}=k T_{0}, D=1 /\left[\Gamma\left(1-\beta_{\Gamma} \mu_{s}^{*}\right)\right]$ is the Doppler factor, and $\eta=D(1-\beta \cos \Phi) /\left[\Gamma\left(1+\beta_{\Gamma} \mu\right)\right] \approx D^{2}\left(1-\mu^{*} \mu_{s}^{*}\right)($ Dermer $\&$ Schlickeiser 1993). The scattered photon spectrum in the $\mathrm{KN}$ region is

$$
\begin{aligned}
\dot{n}_{\mathrm{ph}}^{\mathrm{KN}}\left(\epsilon_{s}^{*}, \mu_{s}^{*}, \tilde{z}\right)= & \frac{D \sigma_{\mathrm{T}} \dot{N}_{0}}{8 \pi^{2}}\left\{\frac{3 D}{8} \frac{n_{e}\left(\epsilon^{*} / D, \mu_{s}^{*}\right)}{\epsilon_{s}^{*}} \frac{m_{e} c^{2}}{E_{0}}\right. \\
& \left.\times \int_{\mu_{\text {min }^{*}}}^{\mu_{\max }^{*}} d \mu^{*} J_{1}\left(\mu^{*}, \tilde{z}\right) J_{2}^{-1}\left(\mu^{*}, \tilde{z}\right) \int_{Y_{\min }}^{\infty} d Y \frac{Y}{\exp (Y)-1} \ln \left[a \epsilon_{s}^{*} J_{2}\left(\mu^{*}, \tilde{z}\right)\left(1-\mu^{*} \mu_{s}^{*}\right) Y\right]\right\},
\end{aligned}
$$

where $a=2 e^{1 / 2}\left(m_{e} c^{2} / E_{0}\right)$ and $Y=\left(m_{e} c^{2} / E_{0}\right)\left[\epsilon / \Gamma J_{2}\left(1-\beta_{\Gamma} \mu^{*}\right]\right.$. The integral limits are as follows:

$$
\begin{aligned}
& Y_{\min }=\frac{m_{e} c^{2}}{\epsilon_{0}} \frac{D^{2}}{\epsilon_{s}^{*}} \frac{1}{J_{2} \eta}, \\
& \mu_{\min }^{*}=\frac{\tilde{z}}{\left(\tilde{z}^{2}+r_{\mathrm{out}}^{2}\right)^{1 / 2}},
\end{aligned}
$$

and

$$
\mu_{\max }^{*}=\frac{\tilde{z}}{\left(\tilde{z}^{2}+r_{\mathrm{in}}^{2}\right)^{1 / 2}} .
$$

The photon spectrum produced by inverse Compton scattering off the electrons at a given $\tilde{z}$ and $\mu_{s}^{*}$ in the anisotropic radiation field from the accretion disk is the sum of equations (23) and (25). Below, the inner and outer radii $r_{\text {in }}$ and $r_{\text {out }}$ of the accretion disk, in units of $10 R_{g}$, are assumed to be 1 and $\infty$, respectively, for our calculations. For the soft photons from the two-temperature accretion disk, putting equation (14) into equation (8) and using equations (B1) and (B2) gives the scattered 
photon spectrum in the Thomson region (see Appendix B),

$$
\begin{aligned}
\dot{n}_{\mathrm{ph}}^{\mathrm{T}}\left(\epsilon^{*}, \mu^{*}, \tilde{z}\right)= & \frac{D \sigma^{\mathrm{T}} \dot{N}_{0}}{8 \pi^{2}} \int_{\max \left[\gamma_{\min }, \epsilon_{s}^{*} / D\right]}^{\gamma_{\max }} \frac{d \gamma}{\gamma^{2}} n_{e}\left(\gamma, \mu_{s}^{*}\right) \\
& \times\left\{1.4 \times 10^{-5} \int_{\mu_{\min ^{*}} \mu_{\mathrm{ho}}^{*}}^{\mu^{*}} \mu^{*}\left(\frac{\epsilon_{s}^{*}}{\eta \gamma^{2}}\right)^{-1.9} \exp \left(-5.93 \frac{\epsilon_{s}^{*}}{\eta \gamma^{2}}\right) J_{3}\left(\mu^{*}, \tilde{z}\right)+\int_{\mu_{\mathrm{ho}}{ }^{*}}^{\mu_{\max }} d \mu^{*} J_{1}\left(\mu^{*}, \tilde{z}\right) \frac{\eta \gamma^{2}}{\epsilon_{s}^{*}} \frac{X^{3}}{\exp X-1}\right\},
\end{aligned}
$$

with

$$
\mu_{\mathrm{ho}}^{*}=\frac{\tilde{z}}{\left(\tilde{z}^{2}+r_{\mathrm{ho}}^{2}\right)^{1 / 2}} .
$$

The scattered photon spectrum in the $\mathrm{KN}$ region is given by

$$
\begin{aligned}
\dot{n}_{\mathrm{ph}}^{\mathrm{KN}}\left(\epsilon^{*}, \mu^{*}, \tilde{z}\right)= & \frac{D \sigma_{\mathrm{T}} \dot{N}_{0}}{8 \pi^{2}} \frac{3 D}{8} \frac{n_{e}\left(\epsilon^{*} / D, \mu_{s}^{*}\right)}{\epsilon_{s}^{*}}\left\{\int_{\mu_{\min ^{*}}}^{\mu_{\mathrm{h}{ }^{*}}} d \mu^{*} J_{3}\left(\mu^{*}, \tilde{z}\right) \int_{\max \left[\epsilon_{1}, \epsilon_{2}\right]}^{\infty} \frac{d \epsilon}{\epsilon}\left[\frac{\epsilon}{\Gamma\left(1-\beta_{\Gamma} \mu^{*}\right)}\right]^{-1.9}\right. \\
& \times \exp \left[-5.93 \frac{\epsilon}{\Gamma\left(1-\beta_{\Gamma} \mu^{*}\right)}\right] \ln \left[\frac{2 e^{1 / 2}\left(1-\mu^{*} \mu_{s}^{*}\right)}{D^{2} \Gamma\left(1-\beta_{\Gamma} \mu^{*}\right)}\right]+\frac{m_{e} c^{2}}{\epsilon_{0}} \int_{\mu_{\mathrm{ho}}{ }^{*}}^{\mu_{\mathrm{max}}} d \mu^{*} J_{1}\left(\mu^{*}, \tilde{z}\right) J_{2}^{-1}\left(\mu^{*}, \tilde{z}\right) \\
& \left.\times \int_{Y_{\min }}^{\infty} d Y \frac{Y}{\exp (Y)-1} \ln \left[a \epsilon_{s}^{*} J_{2}\left(\mu^{*}, \tilde{z}\right)\left(1-\mu^{*} \mu_{s}^{*}\right) Y\right]\right\},
\end{aligned}
$$

respectively, where, $\epsilon_{1}=\epsilon_{0}^{*} / D$ and $\epsilon_{2}=D / \epsilon_{s}^{*}\left(1-\mu \mu_{s}\right) ; J_{1}\left(\mu^{*}, \tilde{z}\right), J_{2}\left(\mu^{*}, \tilde{z}\right)$, and $J_{3}\left(\mu^{*}, \tilde{z}\right)$ are given in equations (B6), (B7), and (B10) of Appendix B. It should be noted that the electron spectrum in the above expressions is determined by equation (21) for a given $\tilde{z}$ and $\mu_{s}^{*}$.

\section{MODEL RESULTS}

If the electrons with power-law distribution are injected at some distance $\tilde{z}_{i}$, then the evolution spectrum of the electrons and the scattered photon spectrum due to inverse Compton scattering at the distance $\tilde{z}$ and scattered angle $\mu_{s}^{*}$ can be calculated by using equations (A11)-(A12) and (B5). Furthermore, we can calculate the instantaneous and stationary spectra of scattered photons, which correspond to local and spatial energy spectra, respectively. According to Dermer \& Schlickeiser (1993), we assume that the blob has volume $V_{b}$ in the jet frame; therefore, the instantaneous and stationary scattered photon spectra can be expressed as

$$
\dot{N}_{\mathrm{ph}}\left(\epsilon_{s}^{*}, \mu_{s}^{*}, \tilde{z}\right)=D V_{b} \dot{n}_{\mathrm{ph}}\left(\epsilon_{s}^{*}, \mu_{s}^{*}, \tilde{z}\right)
$$

and

$$
\dot{N}_{\mathrm{ph}}\left(\epsilon_{s}^{*}, \mu_{s}^{*}\right)=\int_{0}^{\infty} d t^{*} \dot{N}_{\mathrm{ph}}\left(\epsilon_{s}^{*}, \mu_{s}^{*}, \tilde{z}\right),
$$

respectively. The equation of motion for the jet is given by (Dermer \& Schlickeiser 1993)

$$
\tilde{z}\left(t^{*}\right)=\tilde{z}_{i}+\frac{2 \times 10^{-4} \beta_{\Gamma} t^{*}(s)}{M_{8}\left(1-\beta_{\Gamma} \mu_{s}^{*}\right)},
$$

where $t^{*}$ is in units of reception time in the rest frame of the accretion disk. In this section, we will show our model results.

\subsection{Evolution of the Electrons}

Although we have assumed that the injected electron spectrum is isotropic in the rest frame of the jet, the evolving spectrum of the electrons is anisotropic, because the evolution depends on the density number of anisotropic photons. Here we assume that the relativistic electrons are injected at $\tilde{z}_{i}=10$ (i.e., $z_{i}=5 \times 10^{-4} \mathrm{pc}$ if we take $M_{8}=1$ ); the evolving spectrum of the electrons at a given $\tilde{z}$ and $\mu_{s}^{*}$ can be calculated from equation (21). First we calculate the instantaneous spectrum of the electrons with fixed $\mu_{s}^{*}$ by using equation (34). It should be pointed out that the instantaneous spectrum of electrons has same shape as the injected spectrum if $s=2$ (see eq. [21]). As an example, we calculate the evolution of the electron distribution for the soft photons from the standard accretion disk. In Figure $2 a$ we give the instantaneous spectra in five consecutive times and the sum of the instantaneous spectrum (i.e., stationary spectrum) for the injected electron spectrum with $s=2$. It is clear that the instantaneous spectrum has the same shape as that of the injected electron spectrum but a different energy range; however, the stationary electron spectrum is very different from the injected spectrum, i.e., the spectral index is 2 for the injected spectrum and 3 for the stationary spectrum (see eq. [22]). On the other hand, if $s \neq 2$, the instantaneous spectrum will be different from the injected spectrum. For example, the instantaneous spectra with $\theta_{s}^{*}=3^{\circ}$ and $s=3$ are shown in Figure $2 b$. Furthermore, it can be seen from equation (21) that the instantaneous spectra at different scattered angles are different; examples are shown in Figure $2 c$. From Figure $2 c$ we can see that the electron loss decreases with increasing scattering angle.

\subsection{Angular Dependence of Scattered Photon Spectrum}

The scattered photon spectrum can be calculated numerically from equation (B5) once the maximum Lorentz factor of the electrons, mass of the black hole, and mass accretion rate are given. However, it is necessary to estimate the angular 


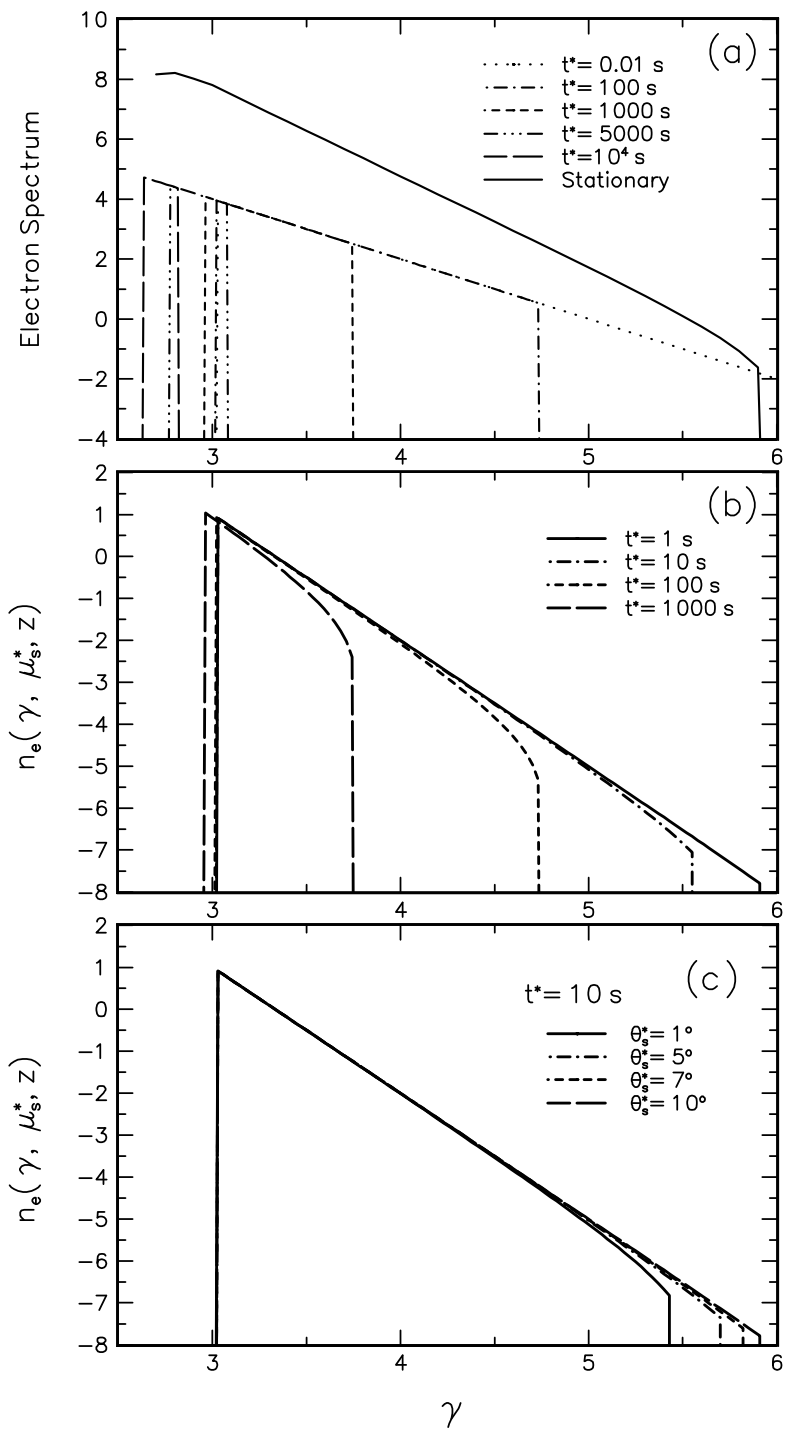

FIG. 2.-Evolution of the electron distribution. (a) Instantaneous evolving electron spectra and stationary electron spectrum for the injected spectral index $s=2$ of the electrons. (b) Instantaneous evolving electron spectra for the injected spectral index $s=3$ of the electrons. (c) Instantaneous evolving electron spectra vs. scatter angle at $t^{*}=10 \mathrm{~s}$. It is assumed that the soft photons come from the standard accretion disk and the parameters used here are $n_{e}^{0}=10^{10}$ $\mathrm{cm}^{-3}, \gamma_{\min }^{i}=10^{3}, \gamma_{\max }^{i}=10^{6}, L_{46}=0.1, z_{i}=5 \times 10^{-4} \mathrm{pc}$, and $\Gamma=10$. The times refer to the rest frame of the accretion disk.

dependence of the scattered photon spectrum. Therefore, we consider the case in which the photons from the standard accretion disk are monochromatic; this case is the same approach as that considered by Dermer \& Schlickeiser (1993). For comparison, here we assume that $s=2$. The photon flux in this case is

$$
\frac{F_{\mathrm{ph}}}{\epsilon^{*}}=\dot{N}_{0} J_{1}\left(\mu^{*}, \tilde{z}\right) \delta\left(\epsilon^{*}-\bar{\epsilon}^{*}\right),
$$

with $\dot{N}_{0}=Q_{0} / m_{e} c^{2}$. In the Thomson region, the photon spectrum is expressed by

$$
\dot{n}_{\mathrm{ph}}^{\mathrm{T}}\left(\epsilon^{*}, \mu^{*}, \tilde{z}\right)=\frac{\sigma_{\mathrm{T}} \dot{N}_{0} n_{e}^{0}}{16 \pi^{2}} D^{s+2}\left(\frac{m_{e} c^{2}}{\epsilon_{0}}\right)^{-(s-1) / 2} \epsilon_{s}^{*-(s-1) / 2} S_{a}^{\mathrm{T}}\left(\mu_{s}^{*}, \tilde{z}\right),
$$

where the angle-dependent function $S_{a}^{\mathrm{T}}$ is

$$
S_{a}^{\mathrm{T}}\left(\mu_{s}^{*}, \tilde{z}\right)=\int_{\mu_{\min }^{*}}^{\mu_{\max }{ }^{*}} J_{1}\left(\mu^{*}, \tilde{z}\right)\left[J_{2}\left(\mu^{*}, \tilde{z}\right) \eta\right]^{(s-1) / 2} d \mu^{*} ;
$$

in the $\mathrm{KN}$ region, the photon spectrum is

$$
\dot{n}_{\mathrm{ph}}^{\mathrm{KN}}\left(\epsilon^{*}, \mu^{*}, \tilde{z}\right)=\frac{\sigma^{\mathrm{T}} \dot{N}_{0} n_{e}^{0}}{8 \pi^{2}} \frac{3}{8} \frac{\epsilon^{*(-s-1)}}{D} S_{a}^{\mathrm{KN}}\left(\mu_{s}^{*}, \tilde{z}\right),
$$




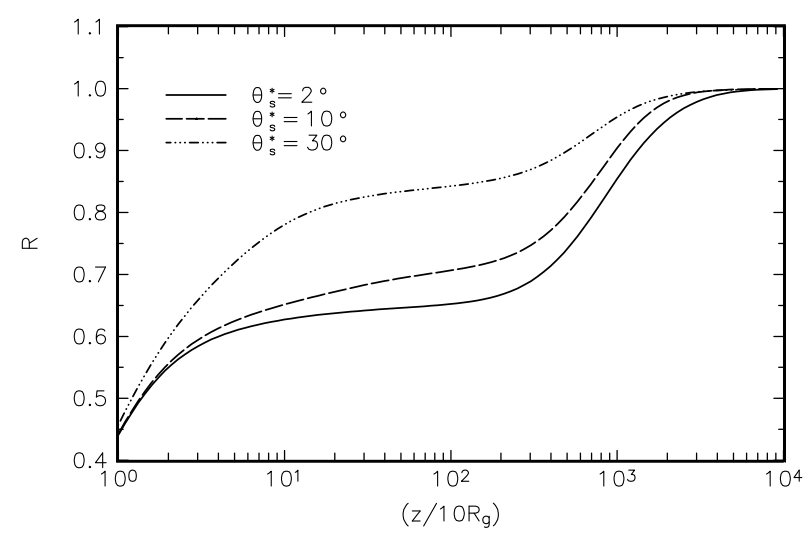

FIG. 3.-Ratio of angle-dependent function in the anisotropic soft photon field from the accretion disk to that given by Dermer \& Schlickeiser (1993) (see text), where three cases for $\theta_{s}^{*}=2^{\circ}, 4^{\circ}$, and $10^{\circ}$ are given.

where the angle-dependent function $S_{a}^{\mathrm{KN}}$ is

$$
S_{a}^{\mathrm{KN}}\left(\mu_{s}^{*}, \tilde{z}\right)=\int_{\mu_{\min ^{*}}}^{\mu_{\max }} \frac{J_{1}\left(\mu^{*}, \tilde{z}\right)}{J_{2}\left(\mu^{*}, \tilde{z}\right) \eta} \ln \left[a \epsilon_{s}^{*} \frac{J_{2}\left(\mu^{*}, \tilde{z}\right) \eta}{D^{2}}\right] d \mu^{*} .
$$

On the other hand, for comparison, we integrate equation (4.8) of Dermer \& Schlickeiser (1993) over the disk radius from inner to outer radii, and after simple algebraic operations, the angle-dependent function in the Thomson region can be given by

$$
S_{i}^{\mathrm{T}}\left(\mu_{s}^{*}, y\right)=\int_{\mu_{\min }^{*}}^{\mu_{\max }} \frac{J_{1}\left(\mu^{*}, y\right)}{\mu^{*}}\left(J_{2} \eta\right)^{(s-1) / 2} d \mu^{*} .
$$

In order to compare $S_{a}^{\mathrm{T}}$ with $S_{i}^{\mathrm{T}}$, the ratio $\mathrm{Ra}=S_{a}^{\mathrm{T}} / S_{i}^{\mathrm{T}}$ is calculated for fixed $s, \mu_{s}^{*}$, and $\tilde{z}$. As an example, the results of Ra versus $\tilde{z}$ for $s=2$ and different $\mu_{s}^{*}$ are shown in Figure 3. From this figure, we see that the inverse Compton spectrum in the anisotropic soft photon field is suppressed in the region of $\tilde{z}<1000$, compared to that in the isotropic photon field. The result calculated from equation (37), for example, is only $60 \%$ of that calculated from equation (40) for $\theta_{s}^{*}=2^{\circ}$ in the region from $\sim 100 R_{g}$ to $\sim 2000 R_{g}$. The difference between equations (37) and (40) is that only a fraction $\mu^{*}$ of the soft photon flux emitted from the surface of the accretion disk should contribute to the inverse Compton scattering.

\subsection{Scattered Photon Spectrum in the Standard Accretion Disk}

Now we turn to the calculation of the inverse Compton spectrum. For the soft photons from the standard accretion disk, the minimum Lorentz factor of the relativistic electrons is between 500 and 1000 . In our calculations, $\gamma_{\min }=1000$ is adopted. Other parameters are chosen as follows: $M_{8}=1, \dot{M}_{26}=0.1$.

For the soft photons from the standard accretion disk, using equations (23)-(28), the instantaneous photon spectra with $\mu_{s}^{*}$ can be calculated for a given height, $z=10 R_{g} \tilde{z}$. In Figure 4, the photon spectra with different $\mu_{s}^{*}$ are shown for $z_{i}=5 \times 10^{-4}$ $\mathrm{pc}, t^{*}=10 \mathrm{~s}$, and $\Gamma=10$. From this figure we see that there is strong beaming of the $\gamma$-ray emission, i.e., the $\gamma$-ray spectrum has its maximum along the jet axis, as pointed out by other authors (e.g., Dermer \& Schlickeiser 1993; Becker \& Kafatos 1995; Zhang \& Cheng 1997). On the other hand, the peak of the instantaneous spectrum will shift down to lower energy as the

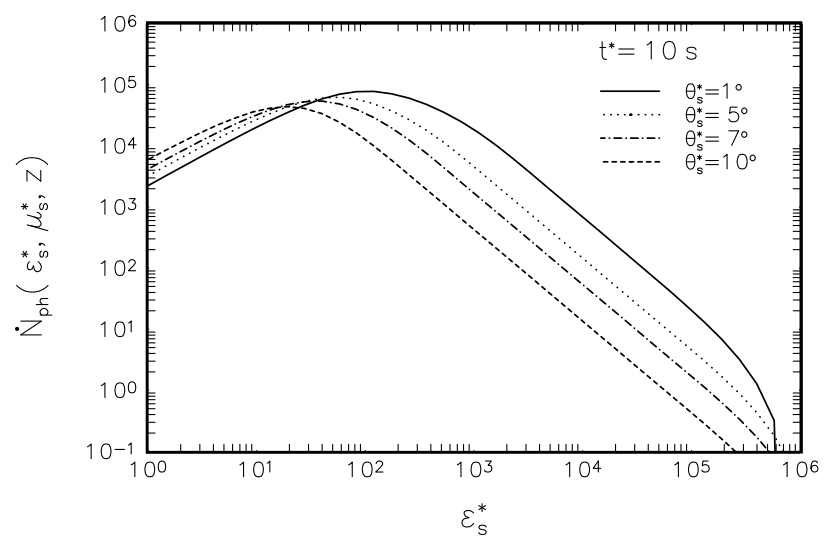

FIG. 4.-Instantaneous $\gamma$-ray spectra with different scatter angles through inverse Compton scattering of the anisotropic photons from the standard accretion disk in the jet. The spectrum is normalized to $\dot{N}_{0}=\sigma_{\mathrm{T}} V_{b} \dot{N}_{0} n_{e 10}^{0} / 4 \pi^{2}$, where $n_{e 10}^{0}=n_{e}^{0} / 10^{10} \mathrm{~cm}^{-3}$, and $V_{b}$ is the volume of the blob. The parameters are the same as in Fig. $2 a$. 
observing angle $\theta_{s}^{*}$ increases. In fact, differentiating equation (23) with respect to $\epsilon_{s}^{*}$, letting it equal zero, and using expression (24), we can obtain the peak energy $\epsilon_{s}^{* \text { peak }}$ of the spectrum as follows:

$$
\epsilon_{s}^{* \text { peak }} \sim \frac{2 E_{0} \gamma^{2}}{m_{e} c^{2}} J_{2}\left(\mu^{*}, \tilde{z}\right) \eta\left(\mu^{*}, \mu_{s}^{*}\right)
$$

This means that $\epsilon_{s}^{* \text { peak }} \propto \eta \cdot \eta \sim 1 /\left(1-\beta_{\Gamma} \mu_{s}^{*}\right)$ for $\mu^{*}=1$, therefore the peak energy shifts down to a lower energy when $\theta_{s}^{*}$ increases.

It is important to investigate the scattered photon spectra at different heights above the accretion disk, which is helpful for understanding the emission region of $\gamma$-rays. We calculated the instantaneous and stationary spectra in the different injection heights. In Figures $5 a-5 c$, the instantaneous photon spectra for different values of the reception time $t^{*}$ (dot-dashed, shortdashed, triple-dot-dashed, and long-dashed lines, for $t^{*}=0.01,100,10^{3}$, and $10^{4} \mathrm{~s}$, respectively in Fig. $5 a$ ) and the stationary photon spectrum (solid line in Fig. $5 a$ ) for $\theta_{s}^{*}=3^{\circ}, s=2$, and $\Gamma=10$, at $z_{i}=2.5 \times 10^{-4} \mathrm{pc}, 5 \times 10^{-4} \mathrm{pc}$, and $5 \times 10^{-3} \mathrm{pc}$ are given. It can be seen from Figures $5 a-5 c$ that the instantaneous spectra are shifted down to a lower energy region as the reception times increase. This feature is due to the evolution of high-energy electrons as the blob flows out. From equation (34), the distance of the blob at time $t^{*}$ can be determined. Obviously, we can define a time $t_{c}^{*}$, corresponding to distance of the blob $z_{c}$; then, the instantaneous spectrum produced at the time which is later than $t_{c}^{*}$ does not contribute to high-energy (e.g., greater than $\mathrm{MeV}) \gamma$-ray radiation for a given injection height $\left(z_{i}\right)$. Therefore we may use $z_{c}-z_{i}$ to describe the size of high-energy $\gamma$-ray emission region. However, $z_{c}-z_{i}$ is about the same order of magnitude as $z_{i}$, because of electron energy loss. Therefore, the larger injection height means a larger emission region. For example, the sizes of emission regions for $\gamma$-rays
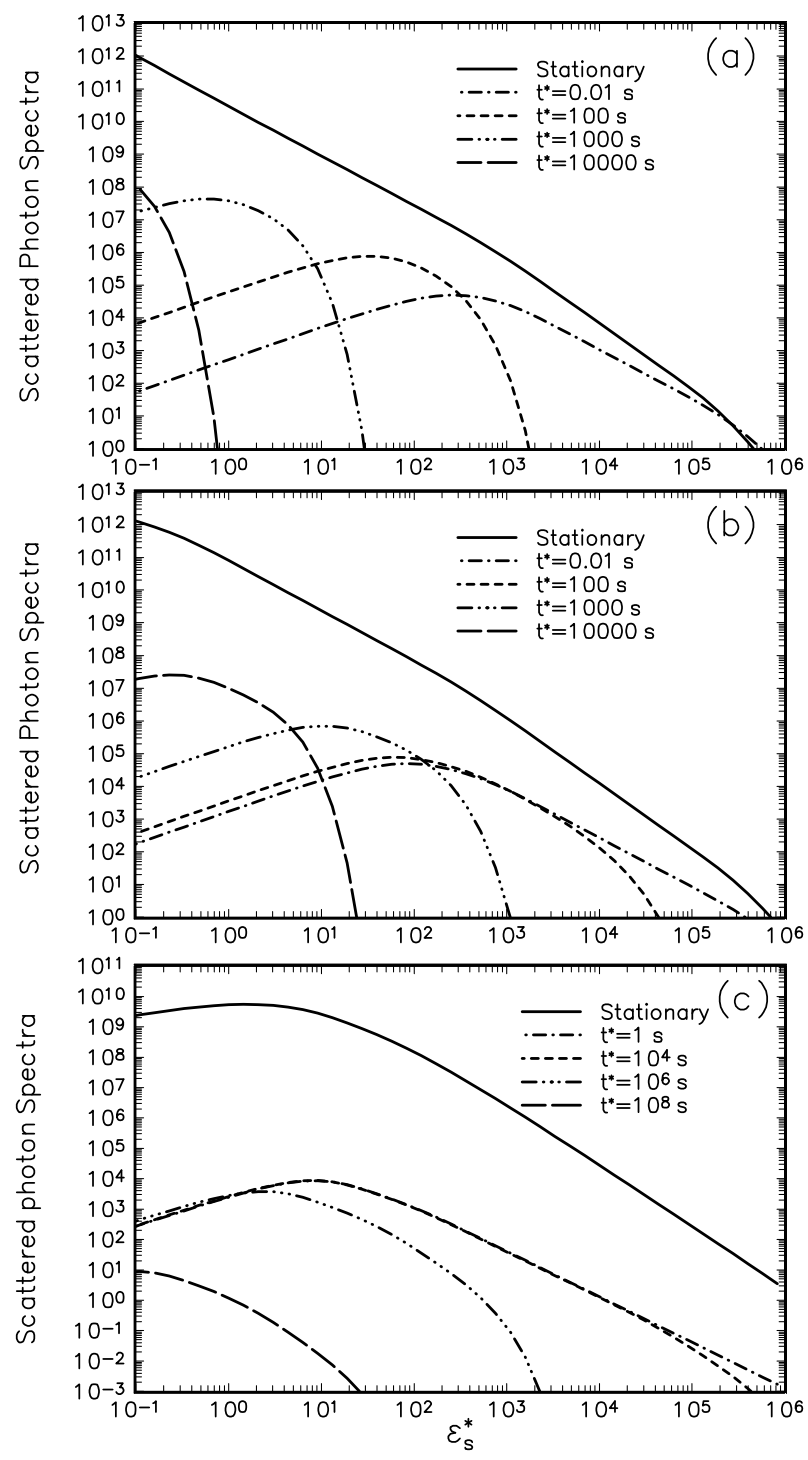

FIG. 5.-Instantaneous and stationary $\gamma$-ray spectra vs. the injected heights of the electrons at the direction of $\theta_{s}^{*}=3^{\circ}$. (a) $z_{i}=2.5 \times 10^{-4}$ pc; $(b)$ $z_{i}=5 \times 10^{-4} \mathrm{pc}$, and $(c) z_{i}=5 \times 10^{-3} \mathrm{pc}$. The soft photons come from the standard accretion disk, and other parameters used here are $n_{e 10}^{0}=1, \gamma_{\min }^{i}=10^{3}$, $\gamma_{\max }^{i}=10^{6}, s=2, L_{46}=0.1$, and $\Gamma=10$. The times refer to the rest frame of the accretion disk. The normalization of the spectrum is the same as that in Fig. 4. 
larger than $\mathrm{MeV}\left(z_{c}-z_{i}\right)$ with injection heights of $z_{i}=2.5 \times 10^{-4}, 5 \times 10^{-4}$, and $5 \times 10^{-3} \mathrm{pc}$ correspond to $10^{-5}, 10^{-4}$, and $10^{-2} \mathrm{pc}$, respectively, for the parameters we used here.

Finally, we calculated the stationary photon spectra in different cases, and the results are shown in Figures $6 a-6 c$. Figure $6 a$ gives the stationary spectra with different $\theta_{s}^{*}$, a particular feature being that $\gamma$-rays are emitted preferentially along the axis of the accretion disk. In Figure $6 b$ the stationary photon spectra at different injection heights $z_{i}$ are shown; it is clear that the shapes of the spectra are different. In Figure $6 c$ the stationary photon spectra at different injection heights $z_{i}$ are shown, which indicate that the jet with high velocity can produce stronger high-energy $\gamma$-ray emission.

\subsection{Scattered Photon Spectrum in the Two-Temperature Accretion Disk}

As mentioned in $\S 3.2$, the minimum Lorentz factor of the electrons is much smaller in the two-temperature accretion disk than that in the standard accretion disk. This results in a very different scattered photon spectrum in the two-temperature accretion disk. Here $\gamma_{\min }=5$ is used, and other parameters are the same as those in $\S 3.2$. The scattered photon spectrum in this case is calculated by using equations (29) and (31).

We have calculated the instantaneous and stationary spectra of the scattered photons in the two-temperature accretion disk. For the instantaneous spectrum, there is also the shift of peak energy, which can be explained approximately by equation (41), but the peak is located at a very low-energy range. The instantaneous and stationary spectra for different injected heights of the relativistic electrons are shown in Figures $7 a-7 c$. It can be seen from these figures that the instantaneous spectrum produced in the height that is close to the injected height of the electrons is an important component of the stationary spectrum, and the emission regions of both X-rays and $\gamma$-rays can be cospatial (i.e., these two emission regions can close each other) because of the low value of the minimum Lorentz factor of the electrons. For the stationary spectrum we calculated the
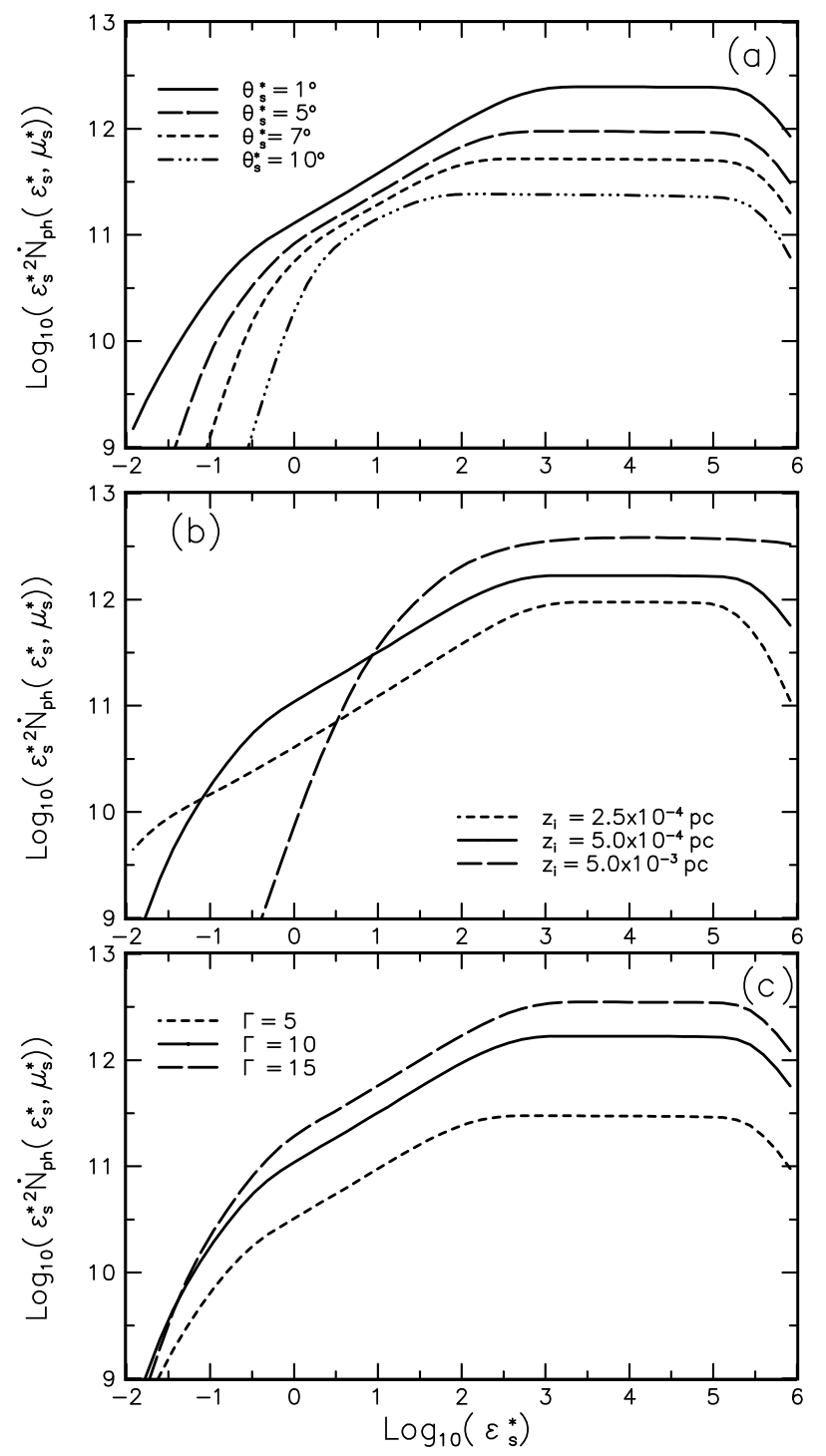

FIG. 6.- Stationary energy spectra $\epsilon_{s}^{* 2} \dot{N}_{\mathrm{ph}}$ of the scattered photons vs. (a) different scattered angles, (b) different injected heights of the relativistic electrons seen at $\theta_{s}^{*}=3^{\circ}$, and $(c)$ different Lorentz factors of the jet seen at $\theta_{s}^{*}=3^{\circ}$. The anisotropic soft photons come from the standard accretion disk, and the relativistic electrons are injected at a height of $z_{i}=5 \times 10^{-4} \mathrm{pc}$. Other parameters are $n_{e 10}^{0}=1, \gamma_{\min }^{i}=10^{3}, \gamma_{\max }^{i}=10^{6}, s=2, L_{46}=0.1$, and $\Gamma=10$. The times refer to the rest frame of the accretion disk. The normalization of the spectrum is same as that in Fig. 4. 

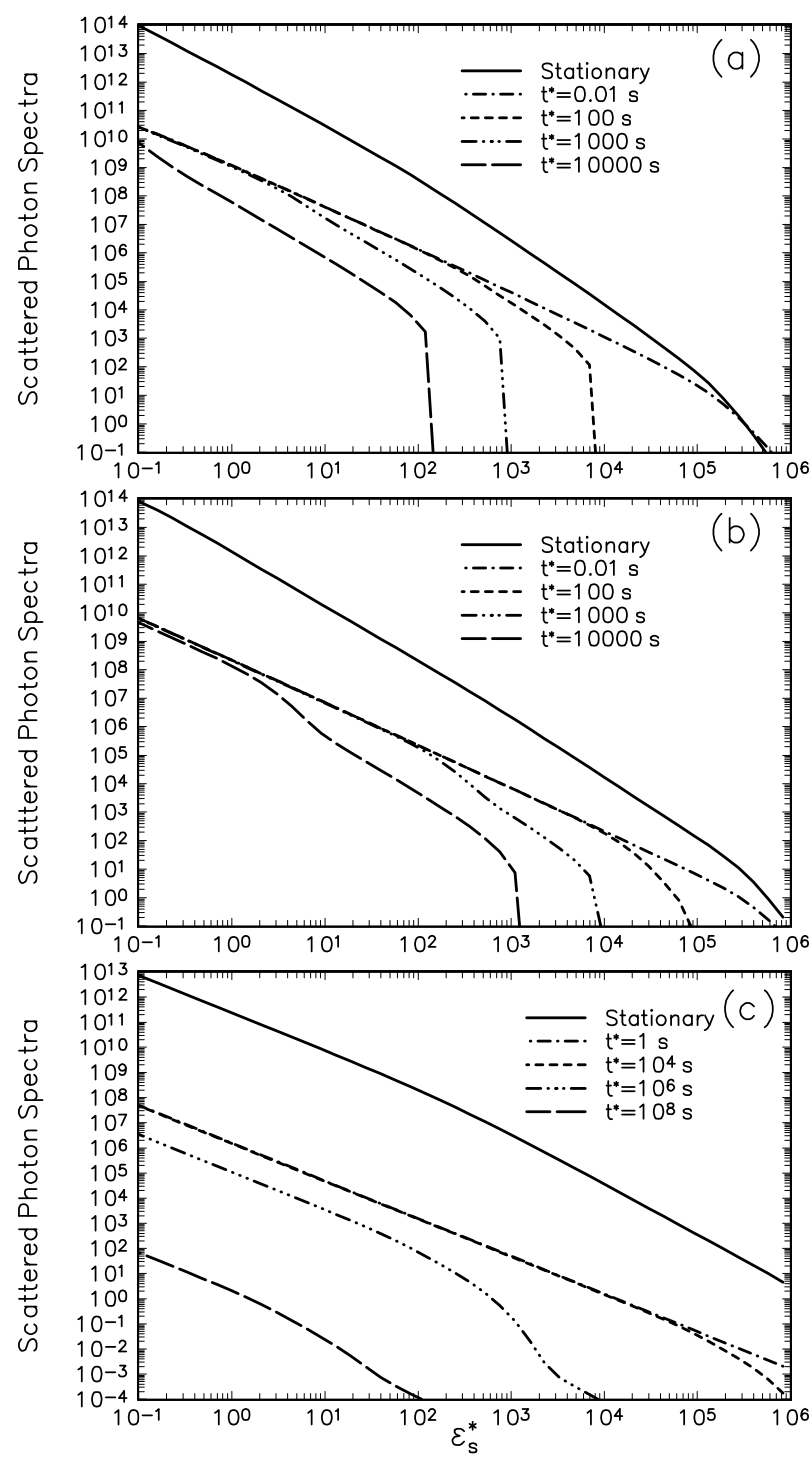

Fig. 7.-Instantaneous and stationary $\gamma$-ray spectra vs. the injected heights of the electrons in the direction of $\theta_{s}^{*}=3^{\circ}$. $(a) z_{i}=2.5 \times 10^{-4}$ pc; $(b)$ $z_{i}=5 \times 10^{-4} \mathrm{pc}$, and $(c) z_{i}=5 \times 10^{-3} \mathrm{pc}$. The soft photons come from the two-temperature accretion disk, and other parameters used here are $n_{e 10}^{0}=1$, $\gamma_{\min }^{i}=10^{3}, \gamma_{\max }^{i}=10^{6}, s=2, L_{46}=0.1$, and $\Gamma=10$. The times refer to the rest frame of the accretion disk. The normalization of the spectrum is the same as that in Fig. 4.

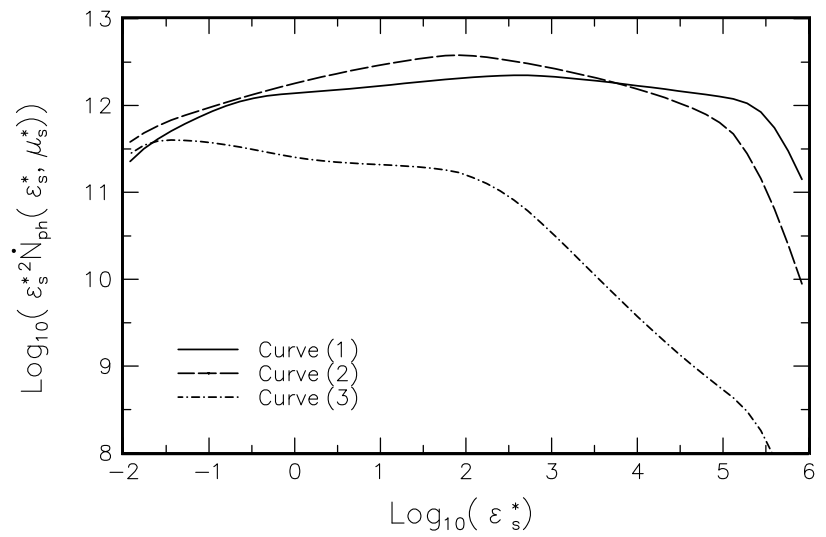

Fig. 8. - Stationary energy spectra $\epsilon_{s}^{* 2} \dot{N}_{\mathrm{ph}}$ of $\gamma$-rays seen at $\theta_{s}^{*}=3^{\circ}$. The anisotropic photons come from the two-temperature accretion disk. Curves (1) and (2) are spectra for heights of $z_{i}=2.5 \times 10^{-4} \mathrm{pc}$ and $z_{i}=5 \times 10^{-4} \mathrm{pc}$, for $s=2$, respectively. Curve (3) is the spectrum at a height of $z_{i}=5 \times 10^{-4} \mathrm{pc}$ for $s=3$, where $n_{e 10}^{0}=10$ is used for comparison. Other parameters are $n_{e 10}^{0}=1, \gamma_{\min }^{i}=5, \gamma_{\max }^{i}=10^{6}, L_{46}=0.1$, and $\Gamma=10$. 
spectra for different spectral indices of injected electrons and injected heights, which are shown in Figure 8. It is clear that the behavior of the spectrum depends on the injected height of the electrons and on the spectral index of the electrons.

\section{DISCUSSION}

Inverse Compton scattering is an important process for $\gamma$-ray production in AGNs. MeV-GeV $\gamma$-rays of AGNs observed by EGRET (Fichtel et al. 1994; von Montigny et al. 1995; Thompson et al. 1995) are produced, it is suggested, by inverse Compton scattering off the relativistic electrons in the jet model (e.g., Maraschi et al. 1992; Dermer \& Schlickeiser 1993; Böttcher et al. 1996). Although the soft photons can have different origins, Böttcher et al. (1996) have shown that the $\mathrm{MeV}-\mathrm{GeV} \gamma$-rays are dominated by inverse Compton scattering of external (standard accretion disk) photons. Apart from the difference of the differential Compton scattering cross sections, their results are the same as our results at large distance. However, according to Bednarek et al. (1996), the anisotropic photon field from the accretion disk may be dominated in the region of $\lesssim 0.1 \mathrm{pc}$ for reasonable parameters. From our calculations, the inverse Compton photon spectrum in the anisotropic soft photon field is slightly suppressed, relative to that in the isotropic soft photon field, and is $\sim 60 \%$ in the emission region from $z=10^{-3}$ to $z=10^{-2} \mathrm{pc}$, so the difference of the amplitudes of the photon spectra for both soft photon fields is not large.

However, a remarkable feature in our calculations is the peak energy shift of the instantaneous scattered photon spectrum, which can indicate the size of the $\gamma$-ray emission region. There are four main factors responsible for this feature: (1) a lower limit of the electron Lorentz factor $\gamma_{\min }$, (2) the Lorentz factor of the jet $\Gamma$ (see eqs. [23], [25], [29], and [31]), (3) the observing angle $\theta_{s}^{*}$ (e.g., Fig. 4), and (4) the distance of $\gamma$-ray emission region to the black hole (Figs. $5 a-5 c$, Figs. $7 a-7 c$ ). In the standard accretion disk, the minimum Lorentz factor of the relativistic electrons produced by $p p$ collision is always much smaller than that produced by $p \gamma$ collision. Therefore, the minimum Lorentz factor of the electrons can be as small as about 12 if $p p$ collision dominates in some region of AGNs, which makes the peak energy of the inverse Compton spectrum shift down to very low energy. If $p \gamma$ collision dominates over $p p$ collision, then the emission regions of $\gamma$-rays and $\mathrm{X}$-rays are not cospatial, because of the larger value of the minimum Lorentz factor of the electrons (see Figs. $5 a-5 c$ ). On the other hand, in the two-temperature accretion disk, the minimum Lorentz factors of the electrons produced by both $p p$ and $p \gamma$ collisions have the same order, which makes the emission regions of both X-rays and $\gamma$-rays cospatial (see Figs. $7 a-7 c$ ). Hence the minimum Lorentz factor of the electrons is a crucial factor in producing X-rays and $\gamma$-rays cospatially. From Figures $5 a-5 c$ and $7 a-7 c$, apart from the possibility of pair production, we can see that if the relativistic electrons are injected in a certain height above the accretion disk, then the $\gamma$-ray emission region can be roughly estimated by using calculated instantaneous spectra. We find that if the injection of the electrons is close to the central powerhouse, the size of the $\gamma$-ray emission region $\left(\sim c t^{*}\right)$ will be smaller. Furthermore, we can use the stationary spectrum to fit the observed spectrum.

The emission region of $\gamma$-rays is usually determined by estimating the optical depth of $\gamma$-rays resulting from pair production. The optical depth of $\gamma$-rays depends on the property of soft photons and has been considered in the isotropic soft field (e.g., Dermer \& Schlickeiser 1994; Blandford \& Levinson 1995) and in the anisotropic soft field (Bednarek 1993; Becker \& Kafatos 1995; Zhang \& Cheng 1997). For the isotropic soft photon field, $\gamma$-rays of $\mathrm{MeV}-\mathrm{GeV}$ can be produced in the region within $\approx 10^{2}-10^{3} R_{\mathrm{g}}$ (Dermer \& Schlickeiser 1994). Furthermore, for an anisotropic soft photon field, the distance of the emission region will be smaller, and the $\gamma$-rays will be focused along the accretion disk because of pair production in the anisotropic soft photon field (Becker \& Kafatos 1995; Zhang \& Cheng 1997). Therefore the optical depth is smaller along the disk axis relative to other directions, and scattered photons produced near the central black hole can more easily escape from the emission region along the disk axis.

We are grateful to the anonymous referee for many detailed comments and useful suggestions. This work is partially supported by a RGC grant of the Hong Kong Government and a CRCG grant of the University of Hong Kong.

\section{APPENDIX A}

\section{THE EQUATION OF MOTION FOR ELECTRONS AND ITS SOLUTION}

The energy loss rate of an electron is determined by the differential cross section of inverse Compton scattering and the scattered photon number density. In the Thomson region, the energy loss rate can be written by using equations (18) and (19),

$$
-\left(\frac{d \gamma}{d t}\right)_{\mathrm{IC}}=\int_{0}^{\infty} d \epsilon_{s} \epsilon_{s} \dot{n}_{\mathrm{ph}}=\frac{\sigma_{\mathrm{T}}}{16 \pi^{2} \Gamma^{2}} \frac{Q_{0}}{m_{e} c^{2}} \gamma^{2} U\left(\mu_{s}, \tilde{z}\right),
$$

with

$$
U=\frac{m_{e} c^{2}}{\pi Q_{0}} \int \frac{d \Omega}{\Gamma^{2}\left(1+\beta_{\Gamma} \mu\right)^{2}} \frac{(1-\beta \cos \Phi)^{2}}{\left(1+\beta_{\Gamma} \mu\right)^{2}} \int_{0}^{\infty} d u F_{\mathrm{ph}}\left[u, \chi\left(\tilde{z}, \mu_{s}\right)\right],
$$

and $u=\Gamma \epsilon_{s}\left(1+\beta_{\Gamma} \mu\right) / \gamma^{2}\left(1-\beta_{\Gamma} \cos \Phi\right)$, where $\cos \Phi$ is given by equation (2), with $\mu_{e}=\mu_{s}$. For simplicity, assuming that $\phi=\phi_{s}^{*}=0$ and then integrating the above expression over $\phi$, we obtain

$$
\int_{0}^{2 \pi}(1-\beta \cos \Phi)^{2} d \phi=\pi\left[2\left(1-\mu \mu_{s}\right)^{2}+\left(1-\mu_{s}^{2}\right)\left(1-\mu^{2}\right)\right]
$$


Furthermore, using $d \mu=\Gamma^{2}\left(1+\beta_{\Gamma} \mu\right)^{2} d \mu^{*}$ and $\mu=\left(\mu^{*}-\beta_{\Gamma}\right) /\left(1-\beta_{\Gamma} \mu^{*}\right)$, the equation (A2) can be written as

$$
U\left(\mu_{s}^{*}, \tilde{z}\right)=\frac{m_{e} c^{2}}{Q_{0}} D^{2} \Gamma^{2} \int d \mu^{*}\left[\left(1-\mu^{*} \mu_{s}^{*}\right)^{2}-\left(1-\mu^{* 2}\right)\left(1-\mu_{s}^{* 2}\right)\right] \int_{0}^{\infty} d u F_{\mathrm{ph}}\left[u, \chi\left(\tilde{z}, \mu_{s}^{*}\right)\right],
$$

where $D=1 /\left[\Gamma\left(1-\beta_{\Gamma} \mu_{s}^{*}\right)\right]$ is the Doppler factor. The equation of motion for a jet at constant velocity $\beta_{\Gamma} c$ is $z\left(t^{*}\right)=z_{i}$ $+\beta_{\Gamma} c t^{*}$, and $d t=\Gamma d t^{*}$, introducing two dimensionless quantities, $\tilde{z} / 10 R_{g}$ and

$$
\xi=\frac{Q_{0}}{m_{e} c^{2}} \frac{\sigma_{\mathrm{T}}}{16 \pi^{2} \Gamma^{3}} \frac{10 R_{g}}{\beta_{\Gamma} c} \approx 3.3 \frac{L_{46}}{\beta_{\Gamma} M_{8}^{2} \Gamma^{3}},
$$

where $L_{46}$ is the luminosity from the accretion disk in units of $10^{46} \mathrm{ergs} \mathrm{s}^{-1}$ and is derived from $L=\int Q(r) 2 \pi R d R$. Hence the equation of motion for an electron for a given angle $\mu_{s}^{*}$ becomes

$$
-\frac{d \gamma}{d \tilde{z}}=\xi U\left(\mu_{s}^{*}, \tilde{z}\right) \gamma^{2} .
$$

In order to solve equation (A6), we need a concrete expression of the differential energy flux of the photons. Now, we consider the case in which soft photons come from the standard accretion disk. From equations (3)-(7) and using $r=$ $\tilde{z}\left(1-\mu^{* 2}\right)^{1 / 2} / \mu^{*}$,

$$
F_{\mathrm{ph}}\left[u, \chi\left(\tilde{z}, \mu^{*}\right)\right] d u=\frac{Q_{0}}{m_{e} c^{2}} \tilde{z}^{-3}\left(1-\mu^{* 2}\right)^{-3 / 2} \mu^{* 3}\left[1-b \tilde{z}^{-1 / 2}\left(1-\mu^{* 2}\right)^{-1 / 4} \mu^{* 1 / 2}\right] \frac{1}{C} \frac{X^{3} d X}{\exp (X)-1},
$$

therefore

$$
U\left(\mu_{s}^{*}, \tilde{z}\right)=\tilde{z}^{-3} D^{2} \Gamma^{2} \int_{\mu_{\min }^{*}(\tilde{z})}^{\mu_{\max }(\tilde{z})} d \mu^{*}\left(1-\mu_{s}^{*} \mu^{*}\right)^{2}\left(1-\mu^{*}\right)^{-3 / 2} \mu^{* 3}\left[1-b \tilde{z}^{-1 / 2}\left(1-\mu^{* 2}\right)^{-1 / 4} \mu^{* 1 / 2}\right],
$$

where $\mu_{\max }^{*}(\tilde{z})=\tilde{z} /\left(\tilde{z}^{2}+1\right)^{1 / 2}, \mu_{\min }^{*}(\tilde{z})=\tilde{z} /\left(\tilde{z}^{2}+r_{\text {out }}^{2}\right)^{1 / 2}$, and $r_{\text {out }}$ is the outer radius of the disk. This integral can be solved analytically if the effect of disk inner radius is ignored [i.e., $I(r)=1$ in eq. (3)], yielding

$U\left(\mu_{s}^{*}, \tilde{z}\right)=\left.\frac{1}{\left(1-\beta_{\Gamma} \mu^{*}\right)^{2}}\left\{\left(E+\frac{1}{E}\right)\left(3-\mu_{s}^{* 2}\right)-2\left[\frac{3 \mu^{*}}{E}-\frac{\mu^{* 3}}{E}-3 \arcsin \left(\mu^{*}\right)\right] \mu_{s}^{*}+\frac{1}{3}\left(4 E+\frac{4}{E}-\frac{\mu^{* 4}}{E}\right)\left(3 \mu_{s}^{* 2}-1\right)\right\}\right|_{\mu_{\min }{ }^{*}} ^{\mu_{\max }^{*}}$,

where $E=\left(1-\mu^{* 2}\right)^{1 / 2}$. If $r_{\text {out }} \gg \tilde{z}$, we have

$$
\begin{aligned}
U\left(\mu_{s}^{*}, \tilde{z}\right)=\frac{1}{\left(1-\beta_{\Gamma} \mu^{*}\right)^{2}}\left\{\frac { 1 } { 3 } \left[\frac{4}{\sqrt{\tilde{z}^{2}+1}}\right.\right. & \left.+4 \sqrt{\tilde{z}^{2}+1}-\frac{\tilde{z}^{4}}{\left(\tilde{z}^{2}+1\right)^{3 / 2}}\right]\left(3 \mu_{s}^{* 2}-1\right)+2\left[\frac{\tilde{z}^{3}}{\tilde{z}^{2}+1}-3 \tilde{z}+\frac{3}{2} \arcsin \left(\frac{\tilde{z}}{\sqrt{\tilde{z}^{2}+1}}\right)\right] \mu_{s}^{*} \\
& \left.+\left(\frac{1}{\sqrt{\tilde{z}^{2}+1}}+\sqrt{\tilde{z}^{2}+1}\right)\left(3-\mu_{s}^{* 2}\right)\right\} .
\end{aligned}
$$

Furthermore, if $\tilde{z} \gg 1$, then $U \approx 2 \tilde{z}\left(1-\mu_{s}^{*}\right)^{2}$, and $d \gamma / d \tilde{z} \approx 2 \xi \gamma^{2} \tilde{z}^{-2}$, which has the same form as that derived by Dermer \& Schlickeiser (1993) in the isotropic photon field. The above results are suitable for the two-temperature accretion disk.

The solution of the motion equation of the electrons can be given by

$$
\gamma^{-1}\left(\mu_{s}^{*}, \tilde{z}\right)=\gamma_{i}^{-1}+\xi V\left(\mu_{s}^{*}, \tilde{z}\right),
$$

where $\gamma_{i}$ is the Lorentz factor of the electrons at the distance $\tilde{z}_{i}$, and

$$
\begin{aligned}
V\left(\mu_{s}^{*}, \tilde{z}\right)= & \int_{\tilde{z}_{i}}^{\tilde{z}} U\left(\mu_{s}^{*}, \tilde{z}\right) d \tilde{z} \approx \frac{1}{\left(1-\beta_{\Gamma} \mu^{*}\right)^{2}}\left\{-\frac{\left(3 \mu_{s}^{* 2}-1\right)}{3}\left(\frac{3}{\sqrt{1+z^{\prime 2}}}+\frac{4}{z^{\prime 2} \sqrt{1+z^{\prime 2}}}\right)\right. \\
& \left.+2 \mu_{s}^{*}\left[\arctan \left(z^{\prime}\right)-\frac{3}{4}\left(1+\frac{1}{z^{\prime 2}}\right) \arcsin \left(\frac{z^{\prime}}{\sqrt{1+z^{\prime 2}}}\right)\right]-\left(3-\mu_{s}^{* 2}\right) \frac{\sqrt{1+z^{\prime 2}}}{z^{\prime 2}}+\left(3-\mu_{s}^{* 2}\right) \frac{1}{z^{\prime 2}}\right\}\left.\right|_{\tilde{z}_{i}} ^{z} .
\end{aligned}
$$

Now we can give the differential electron spectrum at distance $\tilde{z}$. In fact, from the relation between this spectrum and injection electron distribution (eq. [17]), $n_{e}(\gamma) d \gamma=n_{e}\left(\gamma_{i}\right) d \gamma_{i}$, where $\gamma$ and $\gamma_{i}$ are related by equation (A11), we can obtain the solution of the electron motion equation given by equation (21).

\section{APPENDIX B}

\section{THE SCATTERED-PHOTON SPECTRUM}

We treat the inverse Compton scattering off the electrons in Thomson and KN regions, respectively. According to Dermer \& Schlickeiser (1993), the differential cross sections in Thomson and KN regions can be approximated by

$$
\left(\frac{d^{2} \sigma}{d \epsilon_{s} d \Omega_{s}}\right)_{\mathrm{T}} \approx \sigma_{\mathrm{T}} \delta\left[\epsilon_{s}-\gamma^{2} \epsilon(1-\beta \cos \Phi)\right] \delta\left(\Omega_{s}-\Omega_{e}\right),
$$


and

$$
\left(\frac{d^{2} \sigma}{d \epsilon_{s} d \Omega_{s}}\right)_{\mathrm{KN}} \approx \sigma_{\mathrm{KN}}\left(\epsilon^{\prime}\right) \delta\left(\epsilon_{s}-\gamma\right) \delta\left(\Omega_{s}-\Omega_{e}\right),
$$

respectively, where $\sigma_{\mathrm{KN}}\left(\epsilon^{\prime}\right)=\left(3 \sigma_{\mathrm{T}} / 8 \epsilon^{\prime}\right) \ln \left(2 e^{1 / 2} \epsilon^{\prime}\right)$ and $\epsilon^{\prime} \equiv \gamma \epsilon(1-\beta \cos \Phi)$. Dermer \& Schlickeiser (1993) have examined the accuracy of these approximations and proved that they are good approximations. Putting equations (B1) and (B2) into equation (1) and using the relation of $\dot{n}_{\mathrm{ph}}\left(\epsilon_{s}^{*}, \mu_{s}^{*}, \tilde{z}\right)=\left(\epsilon_{s}^{*} / \epsilon_{s}\right) \dot{n}_{\mathrm{ph}}\left(\epsilon_{s}, \mu_{s}, \tilde{z}\right)$, the photon spectra in the Thomson region and in the $\mathrm{KN}$ region in the rest frame of the accretion disk can be expressed by

$$
\dot{n}_{\mathrm{ph}}^{\mathrm{T}}\left(\epsilon_{s}^{*}, \mu_{s}^{*}, \tilde{z}\right)=\frac{D \sigma_{\mathrm{T}}}{4 \pi^{2}} \int_{\gamma_{\min }}^{\gamma_{\max }} d \gamma \gamma^{-2} n_{e}\left(\gamma, \mu_{s}^{*}, \tilde{z}\right) \int_{\mu_{\min }^{*}}^{\mu_{\max }{ }^{*}} d \mu^{*} F_{\mathrm{ph}}\left(\frac{\epsilon_{s}^{*}}{\eta \gamma^{2}}, \tilde{z}\right)\left(\frac{\epsilon_{s}^{*}}{\eta \gamma^{2}}\right)^{-1}
$$

and

$$
\dot{n}_{\mathrm{ph}}^{\mathrm{KN}}\left(\epsilon_{s}^{*}, \mu_{s}^{*}, \tilde{z}\right)=\frac{D \sigma_{\mathrm{T}}}{4 \pi^{2}} \frac{3 D}{8} \frac{n_{e}\left(\epsilon^{*} / D, \mu_{s}^{*}, \tilde{z}\right)}{\epsilon_{s}^{*}} \int_{D / \epsilon_{s}^{*}\left(1-\mu \mu_{s}\right)}^{\infty} d \epsilon \epsilon^{-1} \int_{\mu_{\min ^{*}}}^{\mu_{\max }{ }^{*}} d \mu^{*} \frac{F_{\mathrm{ph}}\left[\epsilon / \eta\left(1-\beta_{\Gamma} \mu^{*}\right), \tilde{z}\right]}{\epsilon / \eta\left(1-\beta_{\Gamma} \mu^{*}\right)} \ln \left[\frac{2 e^{1 / 2}\left(1-\mu^{*} \mu_{s}^{*}\right)}{D^{2} \Gamma\left(1-\beta_{\Gamma} \mu^{*}\right)}\right],
$$

respectively, where $\eta=D(1-\beta \cos \Phi) /\left[\Gamma\left(1+\beta_{\Gamma} \mu\right)\right] \approx D^{2}\left(1-\mu^{*} \mu_{s}^{*}\right)$ (Dermer \& Schlickeiser 1993), $\gamma_{\max }$ and $\gamma_{\min }$ are the maximum and minimum Lorentz factors of the electrons, and $\mu_{\min }^{*}$ and $\mu_{\max }^{*}$ are given by equations (27) and (28). $\mu=$ $\left(\mu^{*}-\beta_{\Gamma}\right) /\left(1-\beta_{\Gamma} \mu^{*}\right)$ has been used to derive equations (B3) and (B5). Therefore, the total photon spectrum is given by

$$
\dot{n}_{\mathrm{ph}}\left(\epsilon_{s}^{*}, \mu_{s}^{*}, \tilde{z}\right)=\dot{n}_{\mathrm{ph}}^{\mathrm{T}}\left(\epsilon_{s}^{*}, \mu_{s}^{*}, \tilde{z}\right)+\dot{n}_{\mathrm{ph}}^{\mathrm{KN}}\left(\epsilon_{s}^{*}, \mu_{s}^{*}, \tilde{z}\right) .
$$

Next, we give the expressions for the scattered photon spectra for different soft photons. For the soft photons from the standard accretion disk, the differential energy flux is given by equation (6). Using $r=\tilde{z} \tan \left(\theta^{*}\right)=\tilde{z}\left(1-\mu^{*}\right)^{1 / 2} / \mu^{*}$ and equation (4), and introducing the following functions to describe the angle-dependent part of $F_{\mathrm{ph}}$,

$$
J_{1}\left(\mu^{*}, \tilde{z}\right)=r^{-9 / 4}\left[1-(0.6 / r)^{1 / 2}\right]=\tilde{z}^{-9 / 4}\left(1-\mu^{* 2}\right)^{-9 / 8} \mu^{* 9 / 4}\left[1-(\tilde{z} / 0.6)^{-1 / 2}\left(1-\mu^{* 2}\right)^{-1 / 4} \mu^{* 1 / 2}\right]^{3 / 4}
$$

and

$$
J_{2}\left(\mu^{*}, \tilde{z}\right)=r^{-3 / 4}\left[1-(0.6 / r)^{1 / 4}\right]=\tilde{z}^{-3 / 4}\left(1-\mu^{* 2}\right)^{-3 / 8} \mu^{* 3 / 4}\left[1-(\tilde{z} / 0.6)^{-1 / 2}\left(1-\mu^{* 2}\right)^{-1 / 4} \mu^{* 1 / 2}\right]^{1 / 4} .
$$

So the differential energy flux (eq. [6]) can be written as

$$
F_{\mathrm{ph}}=F_{0} J_{1}\left(\mu^{*}, \tilde{z}\right) G(X) \mu_{\min }^{*} \leq \mu^{*} \leq \mu_{\max }^{*},
$$

where $G(X)$ is given by equation (5), with

$$
X\left(\mu^{*}, \tilde{z}, \epsilon_{s}^{*}\right)=\frac{\epsilon_{s}^{*}}{\eta \gamma^{2}} \frac{m_{e} c^{2}}{k T(r)}=\frac{m_{e} c^{2}}{E_{0}} \frac{\epsilon_{s}^{*}}{\gamma^{2}} \frac{1}{J_{2}\left(\mu^{*}, \tilde{z}\right) \eta},
$$

where $E_{0}=k T_{0}$. Inserting equation (B8) into equations (B3) and (B5), we can obtain the scattered photon spectra in Thomson and $\mathrm{KN}$ regions for the soft photons from the standard accretion disk, which are given in equations (23) and (25), respectively.

For the soft photons from the two-temperature accretion disk, we further introduce a function $J_{3}$, in addition to $J_{1}$ and $J_{2}$,

$$
J_{3}\left(\mu^{*}, \tilde{z}\right)=\tilde{z}^{-3}\left(1-\mu^{* 2}\right)^{-3 / 2} \mu^{* 3}\left[1-(\tilde{z} / 0.6)^{-1 / 2}\left(1-\mu^{* 2}\right)^{-1 / 4} \mu^{* 1 / 2}\right] .
$$

The differential energy flux (eq. [14]) can now be expressed by

$$
F_{\mathrm{ph}}= \begin{cases}F_{0}^{h}\left(\frac{\epsilon_{s}^{*}}{\eta \gamma^{2}}\right)^{-\alpha} \exp \left(-\frac{m_{e} c^{2}}{k T_{e}} \frac{\epsilon_{s}^{*}}{\eta \gamma^{2}}\right) H\left(\frac{\epsilon_{s}^{*}}{\eta \gamma^{2}}-\epsilon_{0}^{*}\right), & \mu_{\mathrm{min}}^{*} \leq \mu^{*} \leq \mu_{\mathrm{ho}}^{*}, \\ F_{0} J_{1}\left(\mu^{*}, \tilde{z}\right) G(X), & \mu_{\mathrm{ho}}^{*} \leq \mu^{*} \leq \mu_{\mathrm{max}}^{*},\end{cases}
$$

where, $\mu_{\mathrm{min}}^{*}, \mu_{\mathrm{ho}}^{*}$, and $\mu_{\max }^{*}$ are given in equations (27), (30), and (28). Putting equation (B11) into equations (B3) and (B5), we can obtain the scattered photon spectra in Thomson and $\mathrm{KN}$ regions for the soft photons from the two-temperature accretion disk, which are given by equations (29) and (31), respectively.

Atoyan, A. M. 1992, A\&A, 257, 476

Becker, P. A., \& Kafatos, M. 1995, ApJ, 453, 83

Bednarek, W. 1993, A\&A, 278, 307

Bednarek, W., Kirk, J. G., \& Mastichiadis, A. 1996, A\&A, 307, L17

Begelman, M. C., Rudak, B., \& Sikora, M. 1990, ApJ, 362, 38

Blandford R. D., \& Königl, A. 1979, ApJ, 232, 24

Blandford, R. D., \& Levinson, A. 1995, ApJ, 441, 79

Blumenthal, G. R., \& Gould, R. J. 1970, Rev. Mod. Phys., 42, 237

Böttcher, M., Mause, H., \& Schlickeiser, R. 1997, A\&A, in press

Chodorowski, J., Zdziarski, A. A., \& Sikora, M. 1992, ApJ, 400, 181

\section{REFERENCES}

Dermer, C. D. \& Schlickeiser, R. 1993, ApJ, 416, 458 .1994, ApJS, 90, 945

Dermer, C. D., Schlickeiser, R., \& Mastichiadis, A. 1992, A\&A, 256, L27

Eilek, J. A., \& Kafatos, M. 1983, ApJ, 271, 804

Fichtel, C. E., et al. 1994, ApJS, 94, 551

Ghisellini, G., \& Madau, P. 1996, MNRAS, 280, 67

Hua, X.-M., \& Titarchuk, L. 1995, ApJ, 449, 188

Mannheim, K., \& Biermann, P. L. 1989, A\&A, 221, 211

Mannheim, K., \& Schlickeiser, R. 1994, A\&A, 286, 983

Maraschi, L., Ghisellini, G., \& Celotti, A. 1992, ApJ, 397, L5 


\section{ZHANG \& CHENG}

Protheroe, R. J., Mastichiadis, A., \& Dermer, C. D. 1992, Astropart. Phys., 1,113

Punch, M., et al. 1992, Nature, 358, 477

Reynolds, S. P. 1982, ApJ, 256, 38

Rieke, H., \& Weekes, T. C. 1969, ApJ, 155, 429

Rybicki, G. R., \& Lightman, A. P. 1979, Radiative Processes in Astrophysics (New York: McGraw-Hill)

Shakura, N. I., \& Sunyaev, R. A. 1973, A\&A, 24, 337

Shapiro, S. L., Lightman, A. P., \& Eardley, D. M. 1976, ApJ, 204, 187
Sikora, M., Begelman, M. C., \& Rees, M. J. 1994, ApJ, 421153

Sikora, M., et al. 1987, ApJ, 320 L81

Sunyaev, R. A., \& Titarchuk, L. G. 1980, A\&A, 86, 121

Quinn J., et al. 1996, ApJ, 456, L83

Svensson, R. 1994, ApJS, 92, 585

Thompson, D. J., et al. 1995, ApJS, 101, 259

von Montigny, C., et al. 1995, ApJ, 440, 525

Zhang, L., \& Cheng, K. S. 1997, ApJ, 475, 534 